\title{
Operation Principle of Resonant Tunneling THz Oscillator at Fixed Bias Voltages
}

\author{
PEIJI ZHAO ${ }^{\mathrm{a}, *}$, H. L. CUI ${ }^{\mathrm{b}, \dagger}$, D. WOOLARD ${ }^{\mathrm{c}, \ddagger}$ and FLIEX BUOT ${ }^{\mathrm{d}}$ \\ ${ }^{a}$ Department of Electrical and Computer Engineering, North Carolina State University, Raleigh, NC 27695, USA; \\ ${ }^{\mathrm{b}}$ Department of Physics and Engineering Physics, Stevens Institute of Technology, Hoboken, NJ 07030, USA; \\ ${ }^{\mathrm{c}}$ Army Research Office, RTP, NC 27709, USA; ${ }^{\mathrm{d}}$ Naval Research Lab, Washington, D.C. 20375-5000, USA
}

\begin{abstract}
Based on time-dependent numerical simulation of an double barrier quantum well structure, a time-dependent energy coupling model is presented to account for the operational principle of a new kind of resonant tunneling $\mathrm{THz}$ oscillators.
\end{abstract}

Keywords: Resonant tunneling; THz current oscillation; Simulation; Wigner function

Resonant tunneling diodes (RTD) have been studied as high frequency oscillators for many years [1] [9]. Traditionally, RTD's are implemented purely as a negative differential resistance element with one energy storage element, the device capacitance. This approach of extrinsically inducing oscillations will always encounter output power restrictions by external looses and low frequency design constraints. At the beginning of the 90's, Jensen and Buot found that there are intrinsic high frequency $(\mathrm{THz})$ current oscillations in RTD [4]. The current oscillations are independent of external circuit. Their results provide evidence for a possible intrinsic approach to high frequency power generation. However, the causes of the intrinsic current oscillations are not clear [4-6]. It is very important to understand the causes of the oscillations since the they are important to the intrinsic approach of design of the THz RTD oscillators. In this paper, we will explain the operational principle of the intrinsic $\mathrm{THz}$ RTD oscillators, the origin of the intrinsic high frequency current oscillations.

Our explanation of the origin of intrinsic high frequency oscillations is based on our numerical simulation of a RTD. The detailed numerical technique and the RTD structure parameters used in our simulation can be found in our other paper [7].

Our simulated $\mathrm{I}-\mathrm{V}$ characteristics of the structure used in this paper shows all main features of the experimental results: a plateau-like structure and two hysteresis regions (see Refs. [4] and [7]). This result convinces us of the correctness of our numerical calculation.

\footnotetext{
*e-mail:pzhao@eos.ncsu.edu

${ }^{\dagger}$ Corresponding author. Tel.: (201) 216-5637, Fax: (201) 216-5638, e-mail: hcui@stevens-tech.edu

${ }^{\ddagger}$ Tel.: 919-549-4297, Fax: 919-549-4310.
} 
Figure 1 shows the relationship between current density and simulation time. From this graph we know that there is a bias voltage window (BVW) in which the current density is oscillatory. The center bias voltage (CBV) of the bias window for the resonant tunneling structure (RTS) employed in this paper is $0.248 \mathrm{~V}$. At this bias voltage, the current oscillation is harmonic. Simulation results shows if bias voltage is lower
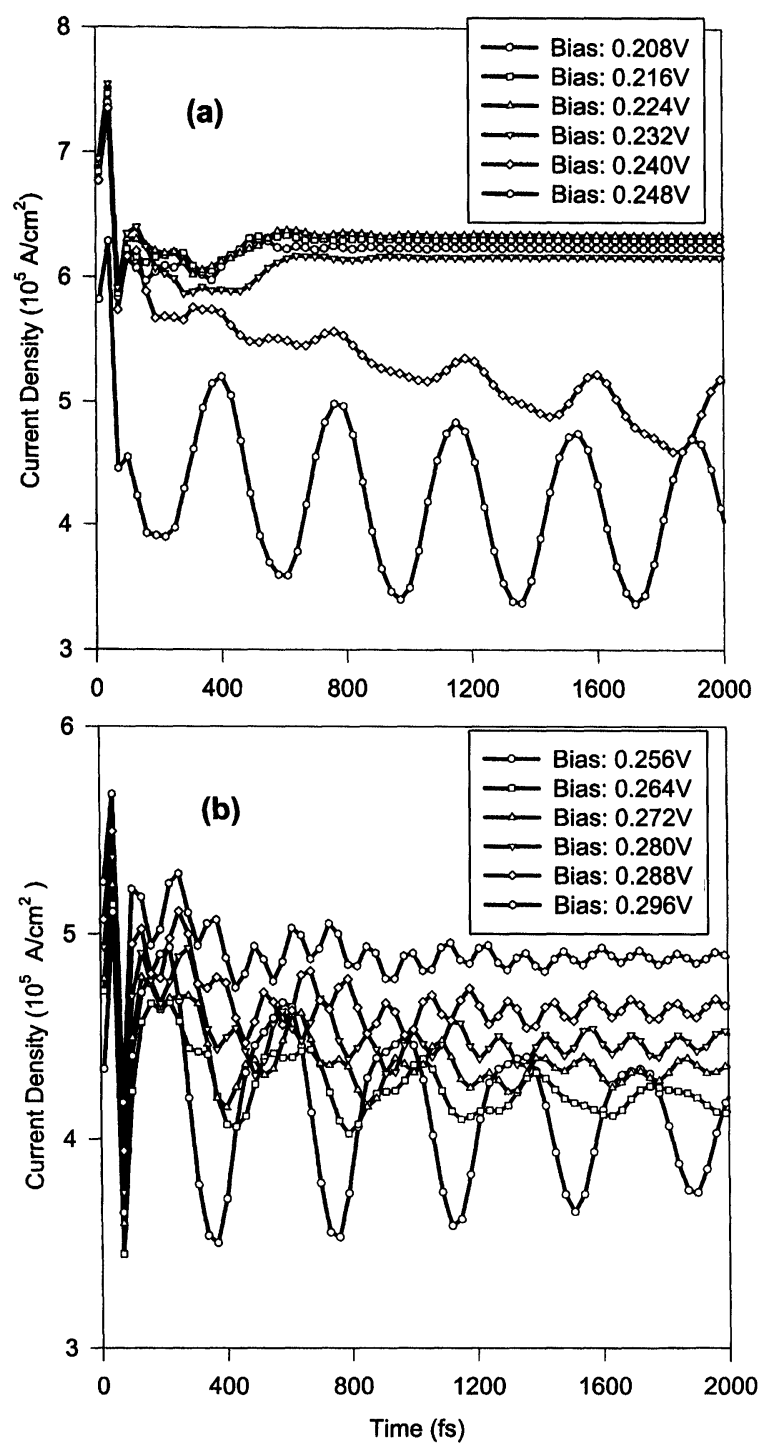

FIGURE 1 Current-time characteristics of the RTD with bias voltages as parameters in the case of forward bias sweep. than the CBV, the current experiences a damping process at first and then develops into a oscillated state. If the bias voltage is greater than the CBV in the BVW, the current densities do not show the initial damping and then harmonic oscillation process that can be observed when bias voltage is less than the CBV. The current gets into a damping process directly with a big initial amplitude. These are the basic features of the current oscillations in the RTS.

Figure $2 \sim$ Figure 4 show time-dependent selfconsistent potential and electron density at special bias voltages. We can see that the current oscillation is concurrent with those of potential and electron density in the whole region of the device. Except the oscillations of potential and density of electron in the whole region of the device, there is emitter quantum well (EQW) in front of the emitter barrier. Of course, the bottom of the EQW oscillates with the increase of the simulation time. Obviously, the current oscillation is related to the creation of an EQW. Figure $2 \sim$ Figure 4 also show that the oscillations have the following features. When the bias just enters the $\mathrm{BVW}$, the potential vibrate a short time before getting into stable oscillation state. The oscillations are periodical rather than irregular. In the $\mathrm{BVW}$, the potential and electron density get into stable oscillation quickly. After the bias voltage get out of the BVW in the higher bias voltage direction, the current oscillation become a decay oscillation, so do the potential and electron density. These situations last until the bias voltage reaches a special point where the plateau-like structure in the $\mathrm{I}-\mathrm{V}$ characteristics ended.

As we have stated, the oscillation of current is closely related to the creation of an EQW, so do those of potential and density distributions. It is very important to note that the EQW, is created just after the current passes the maximum value of the current. Once the current passes the maximum value of the current, the reflection coefficient of the electron-wave increases dramatically. Thus, the interference between the injected electron-wave and the reflected electron-wave leads to depletion 

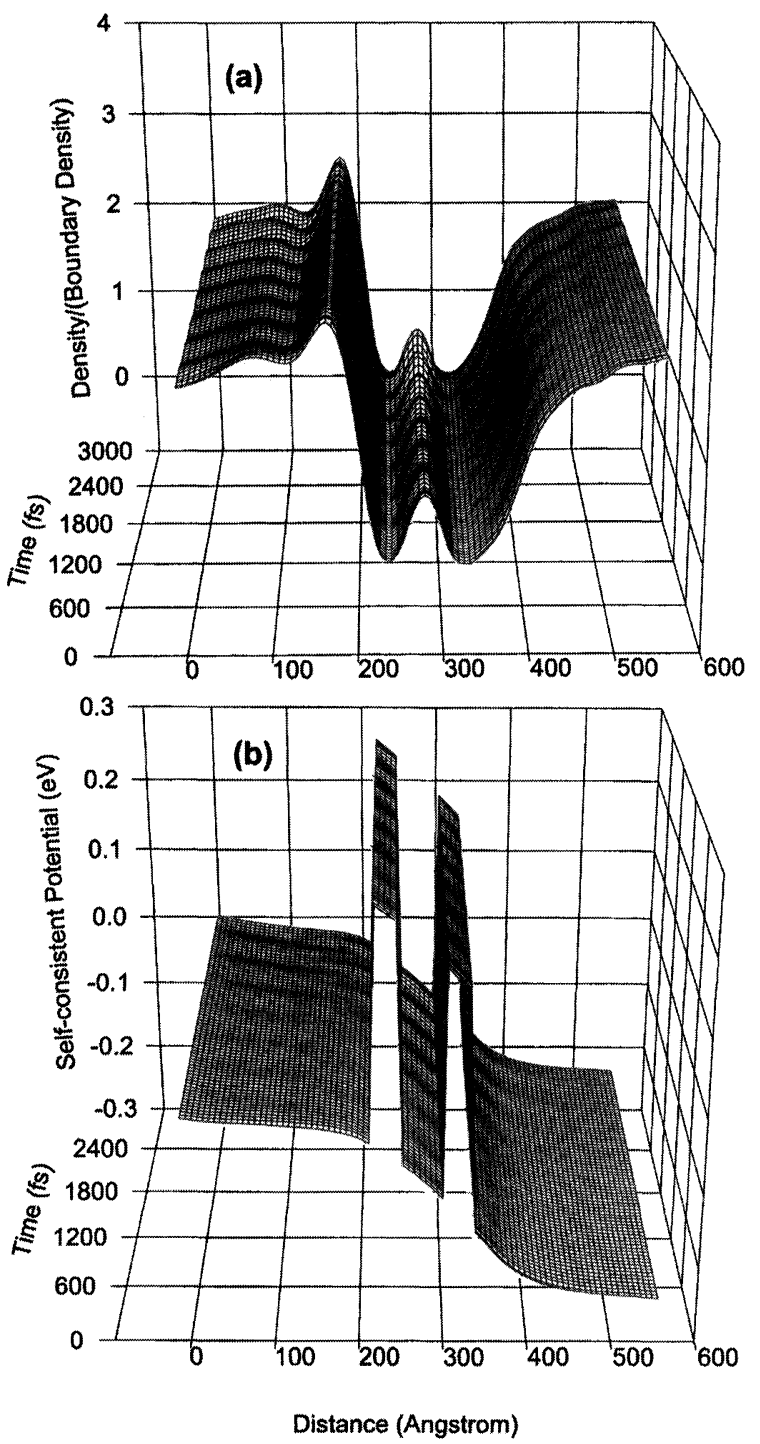

FIGURE 2 Time-dependent electron density distribution and self-consistent potential at bias voltage $0.240 \mathrm{~V}$. The figures show the oscillations of potential and density distribution are getting into stable states. It should be noted that the depth of the EQW is increasing.

of electrons in front of the emitter barrier. The depletion of electrons in front of the emitter barrier further leads to a relatively positive charge background thereby an EQW. Once the EQW is created and the energy level in the EQW is separated from the three dimensional states in the emitter, the coupling between the energy level
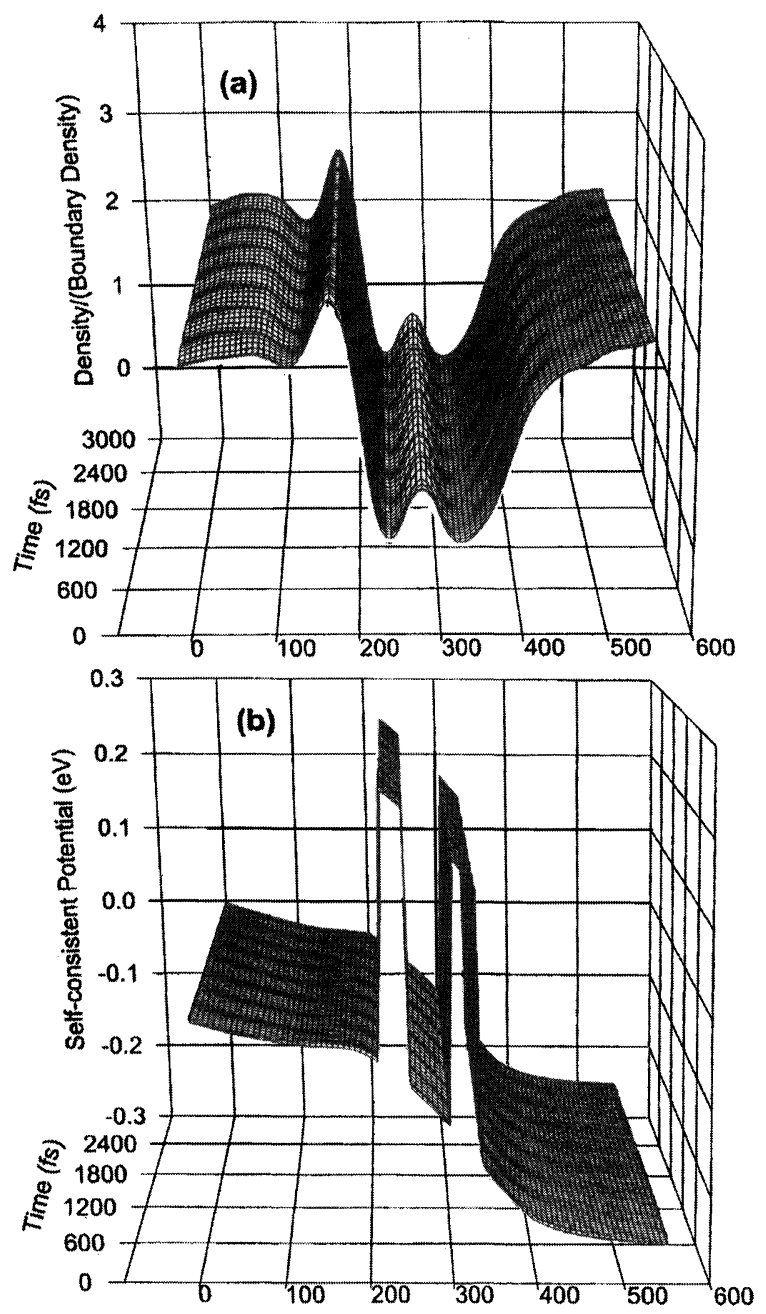

FIGURE 3 Time-dependent electron density distribution and self-consistent potential at bias voltage $0.248 \mathrm{~V}$. The figures show the oscillation of potential and density distribution in stable states. The depth of the EQW is increasing.

in the EQW and that in the main quantum well will causes the oscillation of current through the double barrier system. Suppose that the wavefunctions for the energy levels in the EQW and the main quantum well (MQW) are expressed respectively as

$$
\begin{gathered}
\psi_{\mathrm{EQW}}(z, t)=C_{\mathrm{EQW}}(z, t) e^{i E_{\mathrm{EQW}} t / \hbar} \\
\psi_{\mathrm{MQW}}(z, t)=C_{\mathrm{MQW}}(z, t) e^{i E_{\mathrm{MQW}} t /(\hbar-\gamma t)}
\end{gathered}
$$



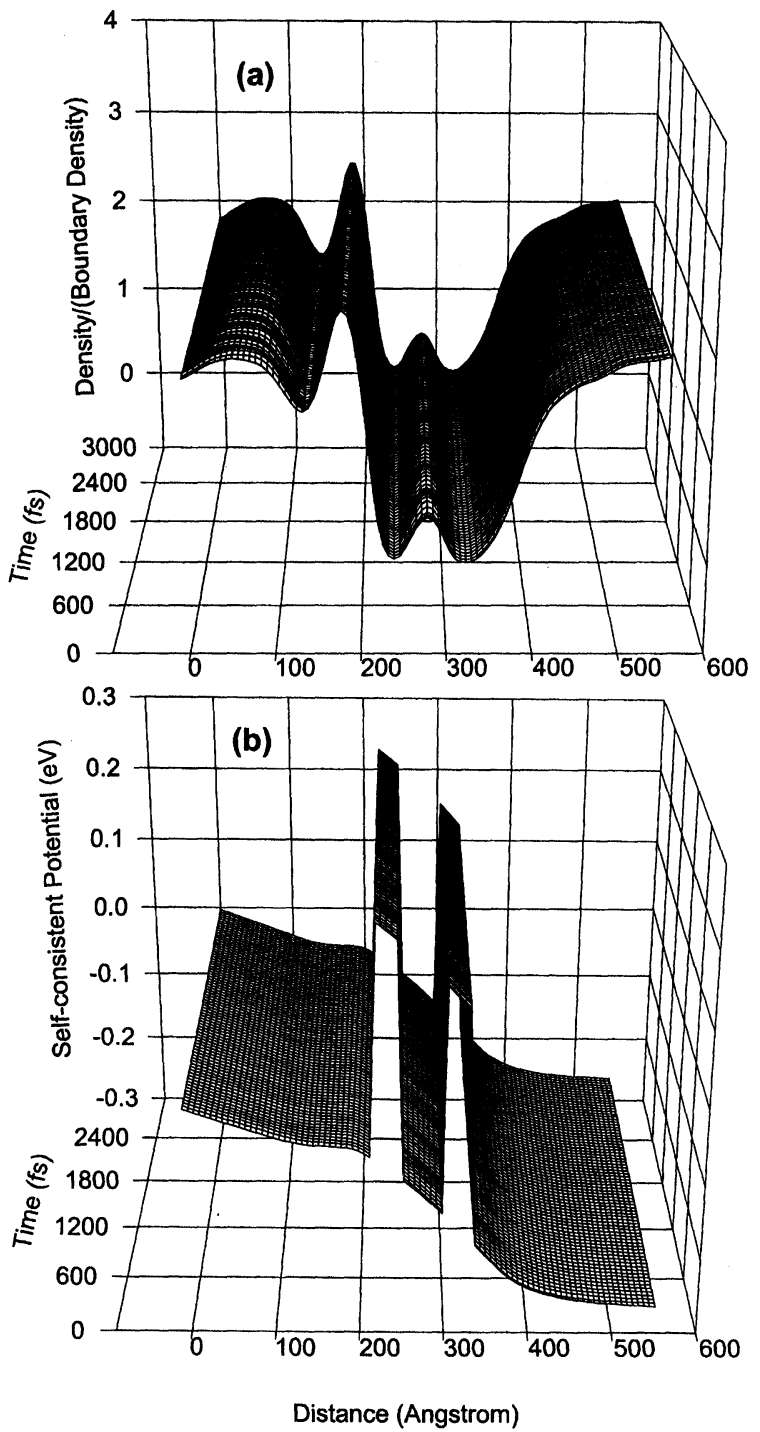

FIGURE 4 Time-dependent electron density distribution and self-consistent potential at bias voltage $0.264 \mathrm{~V}$. The figures show the oscillation of potential and density distribution are damped out. It should be noted that the depth of the EQW is increasing.

In writing the above wave-functions, we have assumed that the width of the energy level in the MQW is much wider than that in the EQW. Considering that the energy level in the MQW is next to the three dimensional states and the energy level in the EQW is a quasi-bounded state, this assumption is a very good one. The coupled state can be expressed by the following wave-function.

$$
\psi(z, t)=C_{1} \psi_{\mathrm{EQW}}(z, t)+C_{2} \psi_{\mathrm{MQW}}(z, t)
$$

With this wave-functions, the current density can be expressed as

$$
\begin{aligned}
&\langle\psi|j| \psi\rangle=\left|C_{1}\right|^{2}\left\langle C_{\mathrm{EQW}}|j| C_{\mathrm{EQW}}\right\rangle \\
&+\left|C_{2} e^{-\gamma t}\right|^{2}\left\langle C_{\mathrm{MQW}}|j| C_{\mathrm{MQW}}\right\rangle \\
&+2 \operatorname{Im}\left(C_{1}^{*} C_{2}\left\langle C_{\mathrm{EQW}}|j| C_{\mathrm{MQW}}\right\rangle\right. \\
&\left.e^{i\left(E_{\mathrm{MQW}}-E_{\mathrm{EQW}}\right) t /(\hbar-\gamma t)}\right) .
\end{aligned}
$$

The electron density can be expressed as

$$
\begin{aligned}
|\psi|^{2}= & \left|C_{1}\right|^{2}\left\langle C_{\mathrm{EQW}} \mid C_{\mathrm{EQW}}\right\rangle \\
& +\left|C_{2} e^{-\gamma t}\right|^{2}\left\langle C_{\mathrm{MQW}} \mid C_{\mathrm{MQW}}\right\rangle \\
& +2 \operatorname{Im}\left(C_{1}^{*} C_{2}\left\langle C_{\mathrm{EQW}} \mid C_{\mathrm{MQW}}\right\rangle\right. \\
& \left.e^{i\left(E_{\mathrm{MQW}}-E_{\mathrm{EQW}}\right) t /(\hbar-\gamma t)}\right)
\end{aligned}
$$

These two equations fully reflect all main features of the simulation results. In Eq. (4), the first term sets the current value at which the oscillation is surrounded at steady states. The second term stands for the damping of the irregularly initial oscillation. The third term represents the oscillation of the current. Separated calculation shows $\Delta E=E_{\mathrm{MQW}}-E_{\mathrm{EQW}} \sim 10 \mathrm{meV}$ in $\mathrm{BVW}$ [8]. Thus, the current oscillation frequency is in the order of $1 \mathrm{THz}$ that coincides with the simulation results shown by Figure 1. In fact, the strength of the oscillations depends on the strength of the coupling between the energy levels and that of the electron-phonon interaction. When bias voltage is small, the factor $e^{-2 \gamma t}$ destroys contributions from the second term in Eq. (4) smoothly. With the increase of the depth of the EQW, the strength of the coupling between the two levels increases. The increase of the coupling leads to the increase of the amplitude of the oscillated currents. If this fact balances the effect of electron-phonon interaction, we get a harmonic current oscillation, 
such as shown by the current oscillation at $0.248 \mathrm{~V}$ in Figure 1. If the strength of electron-phonon interaction is strong enough, the contribution from the second in Eq. (4) gets into effects. This effect is greater at higher bias voltages. Figure 1 confirms our analysis. At bias voltage $0.248 \mathrm{~V}$, the self-consistent potential and electron density distribution all exhibit oscillation behaviors. The oscillation of electron density can be qualitatively explained by Eq. (5).

Our simulation results show that there is only one bias voltage region for the device structure employed in this paper in which the current oscillated. This is due to the electron-phonon interaction that destroys the current oscillations and the exchange of the positions of the energy levels in the EQW and the MQW that leads to the collapse of the EQW. In fact, if the device structure is suitable for the creation of another energy level in the EQW, there will be another bias voltage region in which the current oscillates [9].

\section{CONCLUSION}

We have pointed out the operational principle at a $\mathrm{THz}$ oscillator in an intrinsic approach. The operation of this kind of oscillators is not relied on external circuit but the intrinsic behavior of micro-particles, the wave behavior. The origin of the current oscillation is traced to the development of a dynamic emitter quantum well and the coupling of its energy level with the level of the main quantum well.

\section{Acknowledgments}

The work at Stevens Institute of Technology is supported by the U.S. Office of Naval Research (contract No. N66001-95-M-3472), and by the U.S. Army Research Office (contract No. DAAH0494-G0413 and DAAG55-97-10355).

\section{References}

[1] Mains, R. K. and Haddad, G. I. (1988). Wigner function modeling of resonant tunneling diodes with high peak-tovalley ratios, J. Appl. Phys., 64, 3564.

[2] Sollner, T. C. L. G., Brown, E. R., Goodhue, W. D. and Le, H. Q. (1984). Observation of Millimeter-wave Oscillation from Resonant Tunneling Diodes and some Theorical Consideration of Ultimate Frequency Limits, Appl. Phys. Lett., 50, 332.

[3] Brown, E. R., In: Hot Carriers in Semiconductor Nanostructures (edited by Shah, J.), pp. 469, Academic Press, Boston, 1992.

[4] Jensen, K. L. and Buot, F. A. (1991). Numerical Simulation of Intrinsic Bistability and High-Frequency Current Oscillations in Resonant Tunneling Structures, Phys. Rev. Lett., 66, 1078.

[5] Ricco, B. and Azbel, M. Ya. (1984). Physics of Resonant Tunneling: The One-dimensional Double-barrier Case, Phys. Rev., B29, 1970.

[6] Presilla, C., Jona-Lasinio, G. and Capasso, F. (1991). Nonliner Feedback Oscillation in Resonant Tunneling through Double Barriers, Phys. Rev., B43, 5200.

[7] Peiji Zhao, Cui, H. L. and Woolard, D., Dynamical Instabilities and I-V Characteristics in Resonant Tunneling through Double Barrier Quantum Well Systems, submitted to Phys. Rev. B.

[8] Peiji Zhao, Cui, H. L., Woolard, D., Jensen, K. L. and Buot, F. A. (2000). Numerical Simulation of Resonant Tunneling Structures: Origin of Hysteresis and Plateau-like Structure, J. Appl. Phys., 87, 1337.

[9] Peiji Zhao, Cui, H. L. and Woolard, D., Frequency Tunable Resonant Tunneling $\mathrm{THz}$ Oscillators at Fixed Bias Voltages, submitted to IEEE/Cornell Conference on AdvConcepts in High Speed Semiconductor Devices and Circuits, 2000. 



Submit your manuscripts at

http://www.hindawi.com
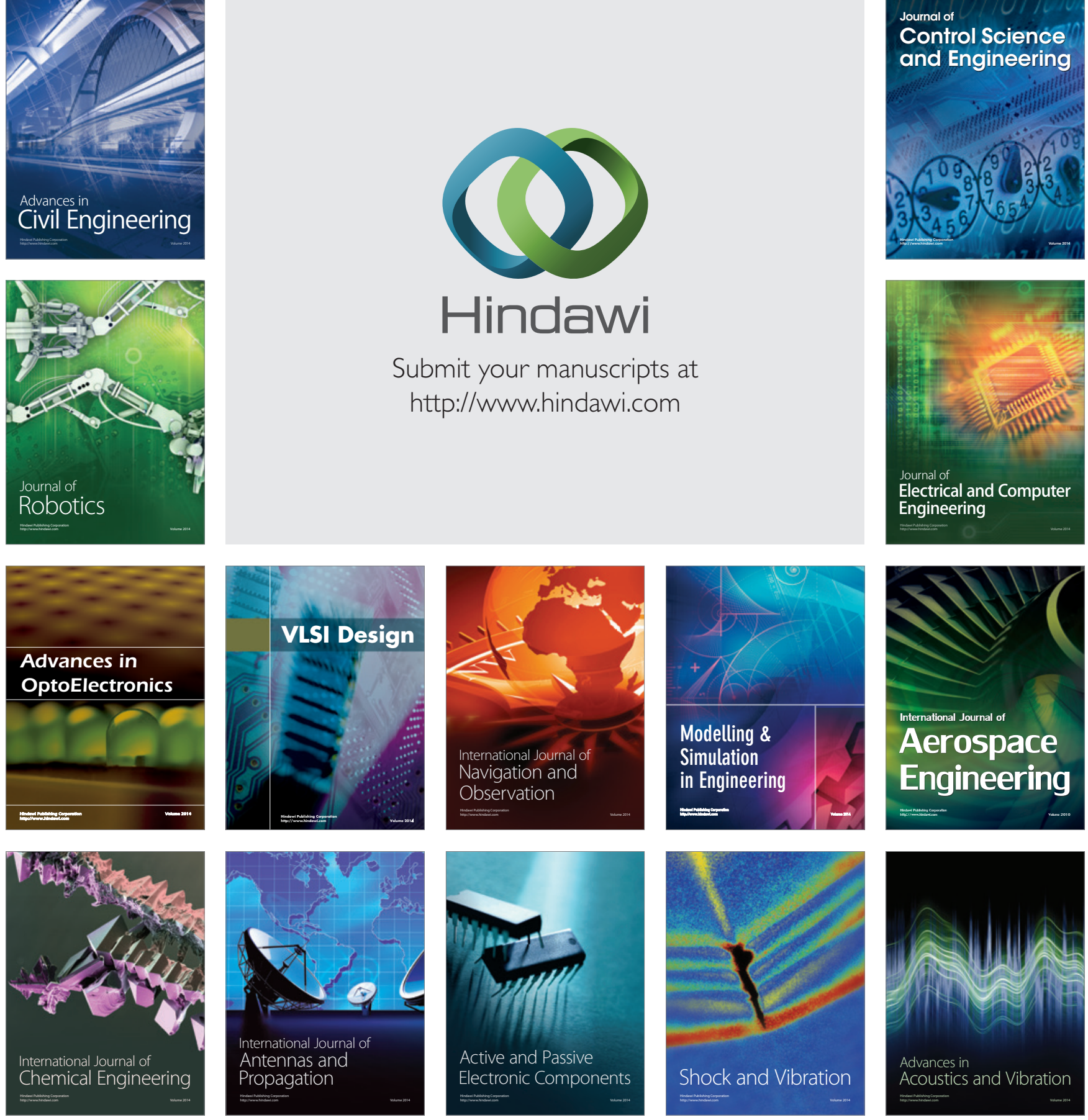